\title{
In vitro activity of tigecycline against clinical isolates of carbapenem resistant Acinetobacter baumannii complex in Pretoria, South Africa
}

\author{
Nahid H Ahmed ${ }^{1 *}$, Kamaldeen Baba ${ }^{1,2}$, Cornelis Clay ${ }^{1}$, Ruth Lekalakala1,2 and Anwar A Hoosen ${ }^{1,2}$
}

\begin{abstract}
Background: The presence of multi-drug resistant Acinetobacter baumannii raises a big therapeutic challenge in our hospital. Tigecycline, a new glycylcycline with expanded broad spectrum of activity against multi-drug resistant organisms was recently licensed in South Africa.

Aim: The aim of this study was to evaluate the in vitro activity of tigecycline against carbapenem resistant A. baumannii complex.

Methods: Consecutive clinical isolates of carbapenem resistant A. baumannii complex were collected between February and July 2010. Species identification and susceptibility testing was performed by Vitek-2 colorimetric compact system with Advanced Expert System (AES). Strains were tested for carbapenemase production by the modified Hodge test, according to the Clinical and Laboratory Standards Institute (CLSI) guidelines.

Results: A total of 232 carbapenem resistant clinical isolates of A. baumannii complex were collected over the six months study period; 217 (93.5\%) of these were modified Hodge test positive. All isolates were susceptible to colistin and 174 (78\%) susceptible to amikacin whilst 20 (9\%) were susceptible to ciprofloxacin. For tigecycline 169 (75.8\%) were fully susceptible, 37 (16.6\%) intermediately resistant and only 17 (7.6\%) were fully resistant. None of the carbapenem resistant isolates were susceptible to ampicillin, amoxicillin/clavullanic acid, piperacillin/tazobactam, cefuroxime, cefuroxime axetil, cefoxitin, cefepime or nitrofurantoin.

Conclusion: All carbapenem resistant isolates were found to be fully susceptible to colistin; amikacin and tigecycline susceptibility was $78 \%$ and $76 \%$ respectively. Treatment options for infections due to carbapenem and multi-drug resistant A. baumannii organisms are limited and hence tigecycline and amikacin may be considered. The properties of tigecycline i.e. stability, safety, low toxicity, non cross-resistance with other antibiotics and its efficacy against multi-drug resistant $A$. baumannii isolates make it a good choice. However, ongoing monitoring of A. baumannii susceptibility to tigecycline is needed.
\end{abstract}

Keywords: Tigecycline, Carbapenems, Acinetobacter baumannii complex

\section{Background}

Glycylcyclines, are novel group of drugs which are tetracycline analogues that circumvent resistant mechanisms against tetracycline [1]. Tigecycline is the first member of the glycylcyclines group to be launched and acts on the ribosomes by inhibiting protein synthesis [2]. Tigecycline shows very good in vitro activity against

\footnotetext{
* Correspondence: nahidhussaan@gmail.com

'Department of Medical Microbiology, Faculty of Health Sciences, University of Pretoria, Prinshof Campus, Pathology Building, 5 Bophelo Road, Private Bag X323, Code: 0007, Riviera, Pretoria 0084, South Africa

Full list of author information is available at the end of the article
}

important nosocomial pathogens such as Escherichia coli, Klebsiella pneumoniae and multi drug resistant (MDR) A. baumannii, it is also active against extended-spectrum beta-lactamase (ESBL) producing strains [1]. Tigecycline does not present cross-resistance with other antibiotics such as $\beta$ lactams or fluoroquinolones [3].

A. baumannii has emerged as an important, troublesome nosocomial pathogen globally. Its clinical significance is due to its ability to easily acquire resistance determinants, making it one of the organisms threatening the currently available therapeutic panel of antimicrobials [4]. Furthermore, 
these organisms have the ability to survive for prolonged periods in the hospital environment, potentiating its ability for nosocomial spread. In the intensive care units (ICU) setting the most vulnerable patients are usually those with ventilator associated pneumonia [4]. The use of carbapenems to treat $A$. baumannii infection has resulted in outbreaks of infection with carbapenem-resistant Acinetobacter species [5].

In recent years in South Africa, we have observed a marked increase in the number of ICU infections due to MDR) A. baumannii (unpublished National Antibiotic Surveillance Forum data). This raises a therapeutic challenge and with the recent licensing in South Africa of tigecycline, a new glycylcycline with expanded broad spectrum of activity against MDR organisms we have an additional option for management of such infections. This study was undertaken to evaluate the in vitro activity of tigecycline against carbapenem resistant $A$. baumannii from patients attending the Steve Biko Academic Hospital Complex in Pretoria.

\section{Results}

During the 6 month period carbapenem resistant $A$. baumannii complex isolates were cultured from 232 patients. Patient information and specimen types are shown in Table 1. Fifty nine percent of patients were males $48.7 \%$, were in the $31-59$ year age group and $62.5 \%$ were from the ICU. The less than 10 year age group accounted for $30(12.9 \%)$ patients.

From the 232 carbapenem resistant A. baumannii complex isolates, a total of $217(93.5 \%)$ were modified Hodge test positive.

Table 1 Patient and specimen information $(\mathbf{N}=\mathbf{2 3 2})$

\begin{tabular}{ll}
\hline Age groups & \\
\hline $\mathbf{3 0}$ years & $87(37.5 \%)$ \\
$\mathbf{3 1 - 5 9}$ years & $113(48.7 \%)$ \\
$\geq \mathbf{6 0}$ years & $32(13.8 \%)$ \\
\hline Gender & $139(59.9 \%)$ \\
\hline Male & $93(40.1 \%)$ \\
\hline Females & \\
\hline Wards & $145(62.5 \%)$ \\
\hline ICUs & $87(37.5 \%)$ \\
\hline Non-ICUs & $149(64.2 \%)$ \\
\hline Specimen type & $20(8.6 \%)$ \\
ETAs* & $15(6.5 \%)$ \\
Blood Culture & $11(4.7 \%)$ \\
CVP tips** & $37(15.9 \%)$ \\
\hline Other**
\end{tabular}

${ }^{*} \mathrm{ETA}=$ Endo-Tracheal Aspirates.

${ }^{*} \mathrm{CVP}=$ Central Venous Puncture.

${ }^{* * *}$ Other $=$ includes wound swabs, tissues and effusions.
Sixty four percent of the specimens were from endotracheal aspirates. Table 2 shows the antimicrobial susceptibility profile of all the carbapenem resistant $A$. baumannii complex isolates. All isolates were susceptible to colistin and $174(78 \%)$ were susceptible to amikacin whilst only 20 (9\%) were susceptible to ciprofloxacin. For tigecycline 169 $(75.8 \%)$ were fully susceptible $(\leq 0.25 \mu \mathrm{g} / \mathrm{ml}), 37(16.6 \%)$ were shown as intermediately resistant $(1-7 \mu \mathrm{g} / \mathrm{ml})$ and only $17(7.6 \%)$ were resistant $(\geq 8 \mu \mathrm{g} / \mathrm{ml})$. None of the carbapenem resistant isolates were susceptible to ampicillin, amoxicillin/clavulanic acid, piperacillin/tazobactam, cefuroxime, cefuroxime axetil, cefoxitin, cefepime and nitrofurantoin.

\section{Discussion}

MDR A. baumannii has emerged as a major cause of hospital acquired infections [5]. Infections caused by these MDR organisms are difficult to treat as only few therapeutic antimicrobial options are available and their eradication from the hospital environment is problematic [6].

Tigecycline, approved by the US Food and Drug Administration (FDA) for the treatment of complicated intra-abdominal and complicated skin and soft tissue infections [6,7], has been shown to have adequate

Table 2 Antimicrobial susceptibility profile* of carbapenem resistant $A$. baumannii complex isolates ( $\mathrm{N}=\mathbf{2 3 2}$ )

\begin{tabular}{|c|c|c|c|}
\hline Antibiotic & $\begin{array}{l}\text { Susceptible } \\
(\mu \mathrm{g} / \mathrm{ml})\end{array}$ & $\begin{array}{l}\text { Intermediate } \\
(\mu \mathrm{g} / \mathrm{ml})\end{array}$ & $\begin{array}{l}\text { Resistant } \\
(\mu \mathrm{g} / \mathrm{ml})\end{array}$ \\
\hline Ampicillin & - & - & $100.0 \%(\geq 32)$ \\
\hline Amoxicillin/clavulanic acid & - & - & $100.0 \% \quad(\geq 32)$ \\
\hline Piperacillin/tazobactam & - & - & $100.0 \%(\geq 32)$ \\
\hline Cefuroxime & - & - & $100.0 \%(\geq 32)$ \\
\hline Cefuroxime Axetil & - & - & $100.0 \%(\geq 32)$ \\
\hline Cefoxitin & - & - & $100.0 \%(\geq 32)$ \\
\hline Cefotaxime & - & - & $100.0 \%(\geq 32)$ \\
\hline Nitrofurantoin & - & - & $100.0 \%(\geq 32)$ \\
\hline Meropenem & - & - & $100.0 \%(\geq 16)$ \\
\hline Imipenem & - & - & $100.0 \%(\geq 16)$ \\
\hline Cefepime & - & $0.4 \%(9-31)$ & $99.6 \%(\geq 32)$ \\
\hline Nalidixic acid & $8.1 \%(\leq 2)$ & - & $91.9 \%(\geq 32)$ \\
\hline Ciprofloxacin & $9.0 \%(\leq 1)$ & - & $91.0 \% \quad(\geq 4)$ \\
\hline $\begin{array}{l}\text { Trimethoprim/ } \\
\text { Sulfamethoxazole }\end{array}$ & $12.6 \%(\leq 20)$ & - & $87.4 \%(\geq 320)$ \\
\hline Ceftazidime & $7.6 \%(\leq 8)$ & $12.6 \%(9-31)$ & $79.8 \% \quad(\geq 64)$ \\
\hline Gentamicin & $9.4 \%(\leq 1)$ & $12.6 \%(2-15)$ & $78.0 \%(\geq 16)$ \\
\hline Amikacin & $78.0 \%(\leq 16)$ & $11.7 \%(17-63)$ & $10.3 \% \quad(\geq 64)$ \\
\hline Tigecycline & $75.8 \%(\leq 0.25)$ & $16.6 \%(1-7)$ & $7.6 \%(\geq 8)$ \\
\hline Colistin & $100.0 \%(\leq 2)$ & - & - \\
\hline
\end{tabular}


activity against a wide variety of microorganisms including A. baumannii. This agent may well become an attractive option for the treatment of infections caused by MDR $A$. baumannii [8].

Tigecycline proved to be safe, well tolerated and effective against a broad spectrum of key community-acquired bacterial pneumonia pathogens, and because it is available in an intravenous (IV) formulation its usage would likely be limited largely to patients requiring hospitalization $[9,10]$. The most frequently reported adverse events with tigecycline were nausea, vomiting, diarrhoea, local IV-site reaction, fever, abdominal pain, and headache [11]. Recent systemic review also found higher mortality with the use of tigecycline making it the last-resort drug in the management of MDR infections [12].

In this study, tigecycline showed very good in vitro activity (MIC $\leq 0.25 \mu \mathrm{g} / \mathrm{ml}$ ) against $75 \%$ of carbapenem resistant $A$. baumannii strains with only $7 \%$ being fully resistant to tigecycline ( $\mathrm{MIC} \geq 8 \mu \mathrm{g} / \mathrm{ml}$ ). This is a slightly lower than what was reported from Taiwan and the USA; where around $85 \%$ of the isolates were susceptible to tigecycline $[13,14]$. However, a recent study from India showed 29\% of MDR Acinetobacters were resistant to tigecycline. This could be due to the fact that tigecycline has been in use in India for several years [15].

In keeping with the report by Molina et al. [16], MDR A. baumannii isolates were also shown to be $100 \%$ susceptible to colistin. However, the issue of nephrotoxicity is a challenge in the management of this infection with an agent such as colistin [17].

All carbapenem resistance isolates were found to be fully susceptible to colistin; amikacin and tigecycline susceptibility was $78 \%$ and $76 \%$ respectively.

The limitation of our study is that it was conducted at the National Health Laboratory Service NHLS, Tshwane district; the laboratory receives specimens for culture and drug susceptibility testing from 5 tertiary-academic, provincial and district hospitals however, our results might not be applicable to other hospitals in Pretoria.

\section{Conclusion}

Treatment options for infections due to carbapenem and multi-drug resistant $A$. baumannii organisms are limited and hence tigecycline and amikacin may be considered options for such infections. The properties of tigecycline i.e. stability, safety, low toxicity, non cross-resistance with other antibiotics and its efficacy against MDR A. baumannii isolates make it a good choice. However, ongoing monitoring of A. baumannii susceptibility to tigecycline is needed.

\section{Material and methods} Study design and sampling

A prospective descriptive study was conducted at the National Health Laboratory Service NHLS, Tshwane district; the laboratory receives specimens for culture and drug susceptibility testing from the Steve Biko Academic Hospital, Kalafong hospital, Tshwane district hospital, Mamelodi hospital, Tshwane metro clinics and the Department of Medical Microbiology University of Pretoria. Consecutive clinical isolates were collected from 232 patients who had carbapenem resistant $A$. baumannii complex infections, over a six month period i.e. between February and July 2010. During this period 539 carbapenem resistant A. baumannii were isolated from the 232 patients. The duplicate isolates from the same patients were excluded for analysis. There was no need for patients consent as the isolates used in this study were not linked to individual patients. This study was approved by the University of Pretoria, faculty of Health Sciences, Ethical committee under this number: (86/2011).

\section{Identification and susceptibility testing}

The VITEK-2 calorimetric compact system with Advanced Expert System (AES), (bioMerieux, France) [18], was used for the identification of isolates and for the antimicrobial susceptibility testing for the carbapenems, tigecycline and other antibiotics by using the using GN identification cards and AST-N133 cards. A. baumannii ATCC BAA 747 was used as a control.

The following antibiotics were tested: imipenem, meropenem, ampicillin, amoxicillin/clavulanic acid, piperacillin/ tazobactam, cefuroxime, cefuroxime axetil, cefoxitin, cefotaxime, ceftazidime, cefepime, amikacin, gentamicin, nalidixic acid, ciprofloxacin, tigecycline, nitrofurantoin, colistin and trimethoprim/sulfamethoxazole. The Clinical and Laboratory Standards Institute (CLSI, 2010) [19] guidelines were used for interpretation.

The modified Hodge test [19] was done to detect the presence of carbapenemases. The test was performed by preparing a $5 \mathrm{ml}$ of $0.5 \mathrm{McF}$ arland standard suspensions from an overnight culture of indicator organism $E$. coli ATCC 25922 and diluted in 1:10, and these were used to swab inoculate the surface of the Muller-Hinton agar plate. After drying the surface, a 10- $\mu$ g ertapenem disk (Becton Dickinson) was placed at the centre, thereafter 3 colonies of test organism grown overnight on a blood agar plate was heavily streaked from the centre (edge of the disc) to the periphery of the plates, then the plate was incubated overnight. The Hodge test is interpreted as positive by the presence of distortion of the inhibition zone.

\section{Competing interest}

The authors declare that they have no competing interests.

\section{Acknowledgments}

The authors are deeply indebted to all the staff at the Department of Medical Microbiology, Faculty of Health Science, University of Pretoria, and the Tshwane Academic Division of the National Health Laboratory Service, South Africa. 


\section{Author details}

'Department of Medical Microbiology, Faculty of Health Sciences, University of Pretoria, Prinshof Campus, Pathology Building, 5 Bophelo Road, Private Bag X323, Code: 0007, Riviera, Pretoria 0084, South Africa. ${ }^{2}$ Tshwane Academic Division of the National Health Laboratory Service, Pretoria, South Africa.

\section{Authors' contribution}

$\mathrm{NA}, \mathrm{KB}$ conceived, participated in the design of the study, carried out the experimental work and participated in drafting the manuscript. AH conceived, participated in the design of the study and participated in drafting the manuscript. CC, RL participated in drafting the manuscript. All authors reviewed and approved the final manuscript.

Received: 23 December 2011 Accepted: 3 May 2012 Published: 3 May 2012

\section{References}

1. Seputiene V, Povilonis J, Armalyte J, Suziedelis K, Pavilonis A, Suziedeliene E: Tigecycline - how powerful is it in the fight against antibiotic-resistant bacteria? Medicina (Kaunas) 2010, 46(4):240-8.

2. European Medicines Agency Tygacil, tigecycline [http://www.ema.europa. eu/ema/index.jsp?]

3. Sorl'ozano A, Guti'errez J, Salmeron A, Luna JD, Martinez-Checa F, Roman J, Piedrola G: Activity of tigecycline against clinical isolates of Staphylococcus aureus and extended-spectrum $\beta$ lactamase-producing Escherichia coli in Granada, Spain. Int J Antimicrob Agents 2006, 28(6):532-6.

4. Peleg AY, Seifert $H$, Paterson DL: Acinetobacter baumannii: Emergence of a Successful Pathogen. Clin Microbiol Rev 2008, 21(3):538-82.

5. Kohlenberg A, Brümmer S, Higgins PG, Sohr D, Piening BC, de Grahl C, Halle E, Ruden $\mathrm{H}$, Seifert H: Outbreak of carbapenem-resistant Acinetobacter baumannii carrying the carbapenemase OXA-23 in a German university medical centre. J Med Microbiol 2009, 58(11):1499-507.

6. Karageorgopoulos DE, Kelesidis T, Kelesidis I, Falagas ME: Tigecycline for the treatment of multidrug-resistant (including carbapenem-resistant) Acinetobacter infections: a review of the scientific evidence. J Antimicrob Chemother 2008, 62(1):45-55.

7. Chen Z, Wu J, Zhang Y, Wei J, Leng X, Bi J, Li R, Yan L, Quan Z, Chen X, Yu Y, Wu Z, Liu D, Ma X, Maroko R, Cooper A: Efficacy and safety of tigecycline monotherapy vs. imipenem/cilastatin in Chinese patients with complicated intra-abdominal infections: a randomized controlled trial. BMC Infect Dis 2010, 21(10):217

8. Liu JW, Wang LS, Cheng YJ, Hsu GJ, Lu PL, Liu YC, Chen CM, Lee CM, Sun W Jang TN, Chiang PC, Chuang YC, Lin HC, Shi ZY, Kung HC, Huang CH, Tsao SM, Lu CT, Liao CH, Hsueh PR: In-vitro activity of tigecycline against clinical isolates of Acinetobacter baumannii in Taiwan. Int J Antimicrob Agents 2008, 32(Suppl 3):188-91.

9. Bergallo C, Jasovich A, Teglia O, Oliva ME, Lentnek A, de Wouters L, Zlocowski JC, Dukart G, Cooper A, Mallick R, 308 Study Group: Safety and efficacy of intravenous tigecycline in treatment of community-acquired pneumonia: results from a double-blind randomized phase 3 comparison study with levofloxacin. Diagn Microbiol Infect Dis 2009, 63(1):52-61.

10. Townsend ML, Pound MW, Drew RH: Potential role of tigecycline in the treatment of community-acquired bacterial pneumonia. Infect Drug Resist 2011, 4:77-86

11. Doan TL, Fung HB, Mehta D, Riska PF: Tigecycline: a glycylcycline antimicrobial agent. Clin Ther 2006, 28(8):1079-106.

12. Yahav D, Lador A, Paul M, Leibovici L: Efficacy and safety of tigecycline: a systematic review and meta-analysis. J Antimicrob Chemother 2011, 66(9):1963-71.

13. Liao CH, Kung HC, Hsu GJ, Lu PL, Liu YC, Chen CM, Lee CM, Sun W, Jang TN, Chiang PC, Cheng YJ, Lin HC, Shi ZY, Wang LS, Chuang YC, Tsao SM, Lu CT, Liu JW, Huang CH, Hsueh PR: In-vitro activity of tigecycline against clinical isolates of Acinetobacter baumannii in Taiwan determined by the broth microdilution and disk diffusion methods. Int J Antimicrob Agents 2008, 32(Suppl 3):192-6.

14. Hoban DJ, Bouchillon SK, Dowzicky MJ: Antimicrobial susceptibility of extended-spectrum beta-lactamase producers and multidrug-resistant Acinetobacter baumannii throughout the United States and comparative in vitro activity of tigecycline, a new glycylcycline antimicrobial. Journal of Diagnostic Microbiology and Infectious Disease 2007, 57(4):423-8.
15. Manoharan A, Chatterjee S, Madhan S, Mathai D: Evaluation of tigecycline activity in clinical isolates among Indian medical centers. Indian Journal of Pathology and Microbiology 2010, 53(4):734-7.

16. Molina J, Cordero E, Pachón J: New information about the polymyxin/ colistin class of antibiotics. Expert Opin Pharmacother 2009, 10(17):2811-28.

17. Chan JD, Graves JA, Dellit TH: Antimicrobial treatment and clinical outcomes of carbapenem-resistant Acinetobacter baumannii ventilator-associated pneumonia. Journal of Intensive Care Medicine 2010, 25(6):343-8.

18. Nakasone I, Kinjo T, Yamane N, Kisanuki K, Shiohira CM: Laboratory-based evaluation of the colorimetric VITEK-2 Compact system for species identification and of the Advanced Expert System for detection of antimicrobial resistances: VITEK-2 Compact system identification and antimicrobial susceptibility testing. Diagn Microbiol Infect Dis 2007, 58(2):191-8.

19. Clinical and Laboratory Standards Institute: Performance standard for antimicrobial susceptibility testing. Twentieth informational supplement. Document M100-S20. Wayne, PA: CLSI; 2010.

doi:10.1186/1756-0500-5-215

Cite this article as: Ahmed et al:: In vitro activity of tigecycline against clinical isolates of carbapenem resistant Acinetobacter baumannii complex in Pretoria, South Africa. BMC Research Notes 2012 5:215.

\section{Submit your next manuscript to BioMed Central and take full advantage of:}

- Convenient online submission

- Thorough peer review

- No space constraints or color figure charges

- Immediate publication on acceptance

- Inclusion in PubMed, CAS, Scopus and Google Scholar

- Research which is freely available for redistribution

Submit your manuscript at www.biomedcentral.com/submit
C Biomed Central 